\title{
SIMPLE PARTIALLY INVARIANT SOLUTIONS
}

\section{S.V. KHABIROV}

\begin{abstract}
The continuous medium models of hydrodynamic type admit 11th dimensional Lie algebra of Galilean group enlarged by uniform dilatation of all independent variables. All subalgebras of this Lie algebra are listed up to inner automorphisms. We consider invariant submodels for subalgebras of the small dimensions from 1 to 3 . For 4 -th dimensional subalgebras, the invariant solutions are the simple solutions depending on finite numbers constants. We formulate a problem on finding partially invariant solutions of the minimal rank. For all 48 types of 4 th dimensional subalgebras we calculate the bases of point invariants in terms of the variables convenient for further calculations. This allows us to consider simplest partially invariant solutions of rank 1 defect 1 . In addition, both regular and irregular partially invariant submodels are obtained.

We consider three of the 4-th dimensional subalgebras producing regular partially invariant solutions in the Cartesian, cylindrical and spherical coordinates, respectively. We obtain a solution depending on an arbitrary function of two variables in Cartesian coordinates. In the cylindrical coordinates, a submodel is reduced to a first order ordinary differential equation. In the spherical coordinates, we generalize invariant solutions of spherical vortex constructed by a rotation group.

We consider two of 4-th dimensional subalgebras producing irregular partially invariant solutions. The arising overdetermined systems are reduced into an involution. The compatibility conditions give a series of exact solutions depending on arbitrary functions, so-called simple waves. We obtain solutions with a level surface of invariant functions in the form of a moving plane with the constant normal but a varying velocity. For stationary motions with a rotation, we obtain the series of exact solutions depending on arbitrary functions.
\end{abstract}

Keyworlds: system of hydrodynamic type, invariants of subalgeba, partial invariant solution, simple solutions.

Mathematics Subjects Classifications: 35B35, 35B06

\section{INTRODUCTION}

Equations of continuous medium mechanics admit Galileo group enlarged by the dilatation. The Lie algebra of this group is 11-dimensional. The group analysis of these equations is to count all subalgebras up to internal automorphisms [1]. For subalgebras of small dimensions from 1 to 3 one considers invariant submodels [2,3,4]. For 4-dimensional subalgebras, the invariant solutions are defined by closed formulae with a bounded number of essential constants. Such solutions are called simple [5]. Solutions of some invariant submodels are simple if the subalgebra of the submodel is embedded into a 4-dimensional overalgebra [6]. Simple solutions can be generalized by considering partially invariant solutions for 4-dimensional subalgebras of the minimal defect [7]. For hydrodynamic type equations, the time $t$ and spatial variables $\vec{x}$ are independent, $\vec{u}$ is the velocity, $p$ is the pressure, $\rho$ is the density. For subalgebras with one invariant in the independent variables, only one function is of general form and we can consider a

S.V. Khabirov, Simple partially invariant solutions,

(C) KHABIROV S.V. 2019.

The work is supported by the federal budjet under the state task 0246-2019-0052 and by RFBR grant 18-2910071.

Submitted October 10, 2018. 
partially invariant submodel of rank 1 and defect 1 . Other subalgebras are associated either with simple invariant or generalized simple irregular partially invariant solutions of rank 1 and defect 1 , which can be reduced to some invariant submodels. The problem is to consider all possible simple partially invariant solutions. The first step is to find the invariants of 4-dimensional subalgebras; there are 48 possible classes [2]. Then by a representation of a partially invariant solution we obtain an overdetermined system of equations of a submodel and the compatibility of this system is to be studied. The compatibility condition determine either a submodel or simple exact solutions.

\section{INVARIANTS OF 4-DIMENSIONAL SUBALGEBRAS}

The model of continuous medium mechanics is symmetric with respect to the transformations in the Galileo group enlarged by the dilatation. This group is associated with an 11-dimensional Lie algebra $L_{11}$ with the basis of first order differentiation operators:

$$
\begin{aligned}
& X_{1}=\partial_{x}, X_{2}=\partial_{y}, X_{3}=\partial_{z}, X_{4}=t \partial_{x}+\partial_{u}, \\
& X_{5}=t \partial_{y}+\partial_{v}, X_{6}=t \partial_{z}+\partial_{w}, X_{7}=y \partial_{z}-z \partial_{y}+v \partial_{w}-w \partial_{v}, \\
& X_{8}=z \partial_{x}-x \partial_{z}+w \partial_{u}-u \partial_{w}, X_{9}=x \partial_{y}-y \partial_{x}+u \partial_{v}-v \partial_{u}, \\
& X_{10}=\partial_{t}, X_{11}=t \partial_{t}+x \partial_{x}+y \partial_{y}+z \partial_{z},
\end{aligned}
$$

where $t$ is the time, $x, y, z$ are Cartesian independent variables, $u, v, w$ are the coordinates of the velocity. Thermodynamical quantities, pressure $p$, density $\rho$, entropy $S$ are related by the state equation $p=f(\rho, S)$ and are invariants of the algebra $L_{11}$. Up to internal automorphisms, there are 48 classes of subalgebras of dimension 4 in $L_{11}[1,2,4]$. It is convenient to calculate the invariants of the subalgebras in special coordinate systems; this provides compact writing of the invariants and simple calculations of submodels. The conventional coordinate systems (CS) are Cartesian coordinates $(D)$, cylindrical coordinates $(C)$ and spherical coordinates $(S)$. Sometimes it is convenient to choose additional change of variables arising in calculating the invariants. The invariants are given in the table. In the first column we provide the number $n . k$ of a subalgebra, where $n$ is the dimension of the subalgebra, $k$ is the number of the subalgebra of the given dimension. In the third column we write the coordinate system, in which the invariants are calculated. The forth column provides the invariants and the fifth shows an additional change of variables. In the second column we provide the basis of subalgebra, where instead of the operators $X_{i}$ we write their subscripts $i$. The constants $a, b$ are invariants of the group of automorphisms.

Let us provide some quantities employed in the table. For the Cartesian coordinate system (D), the symbols $\vec{i}, \vec{j}, \vec{k}$ denote its orthonormal basis,

$$
\vec{x}=x \vec{i}+y \vec{j}+z \vec{k}, \quad \vec{u}=u \vec{i}+v \vec{j}+w \vec{k}, \quad v=q \cos \vartheta_{D}, w=q \sin \vartheta_{D} .
$$

For the cylindrical system $(C)$ we let $y=r \cos \theta_{C}, y=r \sin \theta_{C}$,

$$
\begin{aligned}
& \vec{x}=x \vec{e}_{x}+r \vec{e}_{r}, \quad r \geqslant 0, \quad 0 \leqslant \theta_{C}<2 \pi, \\
& \vec{e}_{x}=\vec{i}, \quad \vec{e}_{r}=\vec{j} \cos \theta_{C}+\vec{k} \sin \theta_{C}, \quad \vec{e}_{\theta_{C}}=-\vec{j} \sin \theta_{C}+\vec{k} \cos \theta_{C}, \\
& \vec{u}=U \vec{e}_{x}+V \vec{e}_{r}+W \vec{e}_{\theta_{C}}, \quad V=q_{C} \cos \vartheta_{C}, \quad W=q_{C} \sin \vartheta_{C}, \quad \vartheta_{D}=\vartheta_{C}+\theta_{C}, \\
& u=U, \quad v=V \cos \theta_{C}-W \sin \theta_{C}, \quad W=V \sin \theta_{C}+W \cos \theta_{C} .
\end{aligned}
$$

For the spherical coordinate system $(S)$, we introduce

$x=r_{S} \sin \theta_{S} \cos \varphi, \quad y=r_{S} \sin \theta_{S} \sin \varphi, \quad z=r_{S} \cos \theta_{S}, \quad r_{S} \geqslant 0, \quad 0 \leqslant \theta_{C} \leqslant \pi, \quad 0 \leqslant \varphi<2 \pi$,

$\vec{x}=r_{S} \vec{e}_{r_{S}}, \quad \vec{u}=U \vec{e}_{r_{S}}+V \vec{e}_{\theta_{S}}+W \vec{e}_{\varphi}, \quad \vec{e}_{r_{S}}=\vec{i} \sin \theta_{S} \cos \varphi+\vec{j} \sin \theta_{S} \sin \varphi+\vec{k} \cos \theta_{S}$,

$\vec{e}_{\theta_{S}}=\vec{i} \cos \theta_{S} \cos \varphi+\vec{j} \cos \theta_{S} \sin \varphi-\vec{k} \sin \theta_{S}, \quad \vec{e}_{\varphi}=-\vec{i} \sin \varphi+\vec{j} \cos \varphi$,

$u=\left(U \sin \theta_{S}+V \cos \theta_{S}\right) \cos \varphi-W \sin \varphi, v=\left(U \sin \theta_{S}+V \cos \theta_{S}\right) \sin \varphi+W \cos \varphi$, 
$w=U \cos \theta_{s}-V \sin \theta_{S}, \quad q_{S}^{2}=|\vec{u}|^{2}=U^{2}+V^{2}+W^{2}, \quad|\vec{x}|=r_{S}, \quad \vec{u} \cdot \vec{x}=q_{S} r_{S} \cos \vartheta_{S}$.

The additional changes (AC) employed in the table are as follows:

$$
\begin{array}{ll}
v=y t^{-1}+\bar{q} \cos \bar{\vartheta}, & w=z t^{-1}+\bar{q} \sin \bar{\vartheta}, \\
v=\frac{z+t y}{1+t^{2}}+\bar{q} \cos \bar{\vartheta}, & w=\frac{z t-y}{1+t^{2}}+\bar{q} \sin \bar{\vartheta} .
\end{array}
$$

\section{Simple REgUlar PARTially INVARIANTS SOLUTIONS}

In the Cartesian system of equations, the hydrodynamics equations are of form $[1,2,4]$

$$
\begin{array}{ll}
u_{t}+u u_{x}+v u_{y}+w u_{z}+\rho^{-1} p_{x}=0, & v_{t}+u v_{x}+v v_{y}+w v_{z}+\rho^{-1} p_{y}=0, \\
w_{t}+u w_{x}+v u_{y}+w w_{z}+\rho^{-1} p_{z}=0, & \rho_{t}+u \rho_{x}+v \rho_{y}+w \rho_{z}+\rho\left(u_{x}+v_{y}+w_{z}\right)=0, \\
S_{t}+u S_{x}+v S_{y}+w S_{z}=0, & p=f(\rho, S) .
\end{array}
$$

The subalgebra 4.48 has invariants (see table), in terms of which, we have the following representation for a regular partially invariant solution of rank 1 defect 1 :

$$
u=u(t, x, y, z), \quad v=v(t), \quad w=w(t), \quad p=p(t), \quad \rho=\rho(t) .
$$

Substituting this representation into system (2.1), we obtain

$$
\begin{aligned}
& v^{\prime}=0, \quad w^{\prime}=0, \quad S^{\prime}=0 \Rightarrow v=v_{0}, \quad w=w_{0}, \quad S=S_{0}, \\
& u_{t}+u u_{x}+v_{0} u_{y}+w_{0} u_{z}=0, \quad \rho u_{x}+\rho^{\prime}=0 \Rightarrow \rho=\frac{\rho_{0}}{1-k t}, \quad u=\frac{-k x+U\left(y-v_{0} t, z-w_{0} t\right)}{1-k t} .
\end{aligned}
$$

The constants $k, v_{0}, w_{0}$ are inessential. They can be removed by the transformations admitted by system (2.1), namely, by the Galilean translations $\left(X_{5}, X_{6}\right)$, a dilatation $\left(X_{11}\right)$ and a translation $\left(X_{10}\right)$. Then we get the solution

$$
u=t^{-1}(x+U(y, z)), \quad \rho=\rho_{0} t^{-1}, \quad p=f\left(\rho_{0} t^{-1}, S_{0}\right),
$$

with an arbitrary function $U(y, z)$.

In the cylindrical coordinates, the hydrodynamics equations read as (the subscript $C$ is omitted)

$$
\begin{aligned}
& D U+\rho^{-1} p_{x}=0, \quad D V+\rho^{-1} p_{r}=r^{-1} W^{2}, \quad D W w+\rho^{-1} r^{-1} p_{\theta}=-r^{-1} V W, \\
& D \rho+\rho\left(U_{x}+V_{r}+r^{-1} W_{\theta}+r^{-1} V\right)=0, \quad D S=0, \quad p=f(\rho, S),
\end{aligned}
$$

where $D=\partial_{t}+U \partial_{x}+V \partial_{r}+r^{-1} W \partial_{\theta}$.

The subalgebra 4.7 has invariants (see table), which give a representation for a regular partially invariant solution $U=U(t, \vec{x})$ of rank 1 defect 1 , where $p, \rho, q, \vartheta$ are functions of the invariant variable $s=r e^{-a \theta}$. We substitute the representation into (2.2):

$$
\begin{aligned}
& U_{t}+U U_{x}+q\left(\cos \vartheta U_{r}+r^{-1} \sin \vartheta U_{\theta}\right)=0, \quad(q \cos \vartheta)^{\prime} q(\cos \vartheta-a \sin \vartheta)+\rho^{-1} p^{\prime}=s^{-1} q^{2} \sin ^{2} \vartheta, \\
& (q \sin \vartheta)^{\prime} q(\cos \vartheta-a \sin \vartheta)-a \rho^{-1} p^{\prime}=-s^{-1} q^{2} \sin \vartheta \cos \vartheta, \\
& \rho^{-1} \rho^{\prime} q(\cos \vartheta-a \sin \vartheta)+U_{x} e^{a \theta}+(q(\cos \vartheta-a \sin \vartheta))^{\prime}+s^{-1} q \cos \vartheta=0, \\
& S^{\prime} q(\cos \vartheta-a \sin \vartheta)=0, \quad p=f(\rho, S) .
\end{aligned}
$$

If $q=0$, we obtain the solution

$$
V=W=0, \quad p=p_{0}, \quad U=U(r, \theta), \quad \rho=\rho(s)
$$

with two arbitrary function. The state equation determines the entropy. 
Table of invariants of 4-dimensional subalgebras

\begin{tabular}{|c|c|c|c|c|}
\hline no. & Basis & CS & Invariants $(p, \rho$ are common $)$ & $\mathrm{AS}$ \\
\hline 4.1 & $7,8,9,11$ & $S$ & $r_{S} t^{-1}, q_{S}, \vartheta_{S}$ & \\
\hline 4.2 & $7,8,9,10$ & $S$ & $r_{S}, q_{S}, \vartheta_{S}$ & \\
\hline 4.3 & $1, a 4+7,10, b 4+11$ & $C$ & $U-a \theta_{C}-b \ln r, q_{C}, \vartheta_{C}$ & \\
\hline 4.4 & $5,6, a 4+7, b 4+11$ & $D$ & $u-x t^{-1}, \bar{q}, a \vartheta-x t^{-1}+b \ln |t|$ & $(1.5)$ \\
\hline 4.5 & $1,4,7,11$ & $C$ & $r t^{-1}, q_{C}, \vartheta_{C}$ & \\
\hline 4.6 & $2,3, a 4+7, b 4+11$ & $D$ & $u-x t^{-1}, q, a \vartheta_{D}-x t^{-1}+b \ln |t|$ & \\
\hline 4.7 & $1,4,10,7+a 11$ & $C$ & $r \exp \left(-a \theta_{C}\right), q_{C}, \vartheta_{C}$ & \\
\hline 4.8 & $2,3,10,7+a 11$ & $C$ & $q_{C}, U, \vartheta_{C}+\theta_{C}-a^{-1} \ln |x|$ & \\
\hline 4.9 & $2,3,7,10$ & $C$ & $x, q_{C}, U$ & \\
\hline 4.10 & $2,3,1+7,10$ & $C$ & $q_{C}, U, \vartheta_{C}+\theta_{C}-x$ & \\
\hline 4.11 & $2,3, a 1+7,4+10$ & $C$ & $q_{C}, U-t, x-2^{-1} t^{2}-a\left(\vartheta_{C}+\theta_{C}\right)$ & \\
\hline 4.12 & $1,4,10,11$ & $D$ & $y z^{-1}, v, w$ & \\
\hline 4.13 & $2,3,10, a 5+11$ & $D$ & $u, v-a \ln |x|, w$ & \\
\hline 4.14 & $2,3,10,11$ & $D$ & $u, v, w$ & \\
\hline 4.15 & $4,5,6,7+a 11$ & $D$ & $\bar{q}, u-x t^{-1}, a \vartheta-\ln |t|$ & $(1.5)$ \\
\hline 4.16 & $4,5,6,7$ & $D$ & $t, \bar{q}, u-x t^{-1}$ & $(1.5)$ \\
\hline 4.17 & $a 1+4,3+5,2-6, b 1+7$ & $D$ & $t, \bar{q}, u-(t+a)^{-1}(x-b \vartheta)$ & $(1.6)$ \\
\hline 4.18 & $a 1+4,5,6, b 1+7, a^{2}+b^{2}=1$ & $D$ & $t, \bar{q}, u-(t+a)^{-1}(x-b \vartheta)$ & $(1.5)$ \\
\hline 4.19 & $1,5,6, b 4+7+a 11$ & $D$ & $\bar{q}, u-b \vartheta, a \vartheta-\ln |t|$ & $(1.5)$ \\
\hline 4.20 & $1,3+5,2-6, a 4+7$ & $D$ & $t, \bar{q}, u-a \vartheta$ & $(1.6)$ \\
\hline 4.21 & $2,3,4,7+a 11$ & $C$ & $q_{C}, U-x t^{-1}, a\left(\vartheta_{C}+\theta_{C}\right)-\ln |t|$ & \\
\hline 4.22 & $2,3,4,1+7$ & $C$ & $t, q_{C}, U-x t^{-1}+t^{-1}\left(\vartheta_{C}+\theta_{C}\right)$ & \\
\hline 4.23 & $1,2,3, b 4+7+a 11$ & $C$ & $q_{C}, U-b a^{-1} \ln |t|, a\left(\vartheta_{C}+\theta_{C}\right)-\ln |t|$ & \\
\hline 4.24 & $1,2,3, a 4+7$ & $C$ & $t, q_{C}, U-a\left(\vartheta_{C}+\theta_{C}\right)$ & \\
\hline 4.25 & $1,2,3, a 4+7+10$ & $C$ & $q_{C}, U-a t, \vartheta_{C}+\theta_{C}-t$ & \\
\hline 4.26 & $4,5,6,11$ & $D$ & $u-x t^{-1}, v-y t^{-1}, w-z t^{-1}$ & \\
\hline 4.27 & $1, a 4+5,6, b 4+11$ & $D$ & $u-a y t^{-1}-b \ln |t|, v-y t^{-1}, w-z t^{-1}$ & \\
\hline 4.28 & $1,5,6, a 4+11$ & $D$ & $u-a \ln |t|, v-y t^{-1}, w-z t^{-1}$ & \\
\hline 4.29 & $1,4,6, a 5+11$ & $D$ & $y t^{-1}-a \ln |t|, v-y t^{-1}, w-z t^{-1}$ & \\
\hline 4.30 & $2,3, a 4+6, b 4+c 5+11$ & $D$ & $u-x t^{-1}, v-c \ln |t|, a w-x t^{-1}+b \ln |t|$ & \\
\hline 4.31 & $2,3,4, a 5+11$ & $D$ & $u-x t^{-1}, v-a \ln |t|, w$ & \\
\hline 4.32 & $2,3,4,11$ & $D$ & $u-x t^{-1}, v, w$ & \\
\hline 4.33 & $1,2,3, a 4+11$ & $D$ & $u-a \ln |t|, v, w$ & \\
\hline 4.34 & $1,2,3,11$ & $D$ & $u, v, w$ & \\
\hline 4.35 & $2,3, a 1+5,4+b 6+10$ & $D$ & $u-t, a v-x+2^{-1} t^{2}, w-b t$ & \\
\hline 4.36 & $2,3, a 1+5,6+10$ & $D$ & $u, a v-x, w-t$ & \\
\hline 4.37 & $2,3,1+5,10$ & $D$ & $u, v-x, w$ & \\
\hline 4.38 & $2,3,5,10$ & $D$ & $x, u, w$ & \\
\hline 4.39 & $1,2,3,4+10$ & $D$ & $u-t, v, w$ & \\
\hline 4.40 & $1,2,3,10$ & $D$ & $u, v, w$ & \\
\hline 4.41 & $\begin{array}{l}1, a 2+b 3+4, c 3+5 \\
d 2+6, a^{2}+b^{2}+(c+d)^{2}=1\end{array}$ & $D$ & $\begin{array}{c}t,(v+(b t-a c) u+c y-t z)\left(t^{2}+c d\right)^{-1} \\
w+((a t+b d) u+a z-t y)\left(t^{2}+c d\right)^{-1}\end{array}$ & \\
\hline 4.42 & $1,4,3+5,2-6$ & $D$ & $t, v-(z+t y)\left(t^{2}+1\right)^{-1}, w+(y-t z)\left(t^{2}+1\right)^{-1}$ & \\
\hline 4.43 & $1,4,5,6$ & $D$ & $t, v-y t^{-1}, w-z t^{-1}$ & \\
\hline 4.44 & $2,1+a 3,3+5,6$ & $D$ & $t, u, v+a x-z+t w$ & \\
\hline 4.45 & $2,3,1+5,6$ & $D$ & $t, u, v-x$ & \\
\hline 4.46 & $2,3,5,6$ & $D$ & $t, x, u$ & \\
\hline 4.47 & $1+a 3,2,5,6$ & $D$ & $t, u, w+(a x-z) t^{-1}$ & \\
\hline 4.48 & $1,2,3,4$ & $D$ & $t, v, w$ & \\
\hline
\end{tabular}


If $\cot \vartheta=a$, then $a=0, \vartheta=\pi / 2, p^{\prime}=\rho r^{-1} q^{2}, U=U(r \theta-t q, r)$, where $q(r), \rho(r)$ are arbitrary functions.

Let us consider the case $q \neq 0, \cot \vartheta \neq a$; in this case $S=S_{0}$ is constant. Equations (2.3) imply the Bernoulli integral

$$
V^{2}+W^{2}+2 i(\rho)=B^{2}
$$

where $i=\int \rho^{-1} d p$ is the enthalpy, and a closed system of the equations of the submodel:

$$
s(a V+W)^{\prime}+W=0, \quad G^{\prime}(V-a W)=G\left(G+a s^{-1} W\right),
$$

where $G=\rho^{-1} \rho^{\prime}(V-a W)+(V-a W)^{\prime}+s^{-1} V$. The function $U$ is determined by the identities

$$
U=-G x e^{-a \theta}+\tilde{U}(t, r, \theta), \quad \tilde{U}_{t}+V \tilde{U}_{r}+r^{-1} W \tilde{U}_{\theta}=\tilde{U} G e^{-a \theta} .
$$

As $a=0$, system (2.4) is reduced to a single equation

$$
\rho^{-1} \rho^{\prime}\left(B^{2}-C^{2} s^{-2}-2 i-f_{\rho}\right)=s \rho\left(B^{2}-C^{2} s^{-2}-2 i\right)-C^{2} s^{-3} .
$$

In spherical coordinates system, hydrodynamics equations read as (we omit the symbol $S$ ):

$$
\begin{aligned}
& D U+\rho^{-1} p_{r}=r^{-1}\left(V^{2}+W^{2}\right), \quad D V+(r \rho)^{-1} p_{\theta}=r^{-1}\left(-U V+W^{2} \cot \theta\right), \\
& D W+(r \rho \sin \theta)^{-1} p_{\varphi}=-r^{-1} W(U+V \cot \theta), \\
& D \rho+\rho\left(U_{r}+r^{-1} V_{\theta}+(r \sin \theta)^{-1} W_{\varphi}+r^{-1} V_{\theta}+2 r^{-1} U+r^{-1} \cot \theta V\right)=0, \\
& D S=0, \quad p=f(\rho, S),
\end{aligned}
$$

where $D=\partial_{t}+U \partial_{r}+r^{-1} V \partial_{\theta}+(r \sin \theta)^{-1} W \partial_{\varphi}$. The subalgebra 4.2 has invariants (see table) determining the representation of a regular partially invariant solution of rank 1 defect 1 :

$$
U=q \cos \vartheta, \quad H=q \sin \vartheta, \quad V=H \cos \lambda, \quad W=H \sin \lambda, \quad \lambda=\lambda(t, \vec{x}),
$$

where $p, \rho, q, \vartheta, U, H$ are functions of one variable $r$. We substitute this representation into (2.5) to obtain:

$$
\begin{aligned}
& U U^{\prime}+\rho^{-1} p^{\prime}=r^{-1} H^{2}, \quad U(r H)^{\prime}=0, \quad U S^{\prime}=0, \quad p=f(\rho, S), \\
& r H^{-1}\left(\lambda_{t}+U \lambda_{r}\right)+\cos \lambda \lambda_{\theta}+\frac{\sin \lambda}{\sin \theta} \lambda_{\varphi}+\sin \lambda \cot \theta=0, \\
& -\sin \lambda \lambda_{\theta}+\frac{\cos \lambda}{\sin \theta} \lambda \varphi+\cot \theta \cos \lambda+r U H^{-1}\left(\ln \left(\rho U r^{2}\right)^{\prime}=0 .\right.
\end{aligned}
$$

If $U=0, H \neq 0$, system (2.7) is incompatible. If $U \neq 0, H=0$, we obtain a generalized radial motion defined by the integrals

$$
S=S_{0}, \quad \rho U r^{2}=C, \quad U^{2}+2 \int \rho^{-1} d p=B^{2}, \quad \lambda=\lambda\left(t-\int U^{-1} d r\right),
$$

where $S_{0}, C, B$ are constants, $\lambda(s)$ is an arbitrary function. As $U \neq 0, H \neq 0$, the integrals

$$
S=S_{0}, \quad H r=H_{0}, \quad U^{2}+\int \rho^{-1} d p+H_{0} r^{-2}=B^{2}
$$

hold. The compatibility condition of system $(2.7)$

$$
r^{2} U H_{0}^{-1} h^{\prime}=h^{2}+1, \quad h=r^{2} U H_{0}^{-1}\left(\ln \left(\rho U r^{2}\right)\right)^{\prime}
$$

defines the submodel. The behavior of integral curves was studied in [8].

In new variables $I=t-\int U^{-1} d r, h \tan n, n^{\prime}=H_{0} r^{-2} U^{-1}$, overdetermined system (2.7) is in involution and becomes

$$
\begin{aligned}
& \lambda_{\theta}+\cos \lambda \lambda_{n}=\tan n \sin \lambda, \\
& \lambda_{\varphi}+\lambda_{n} \sin \lambda \sin \theta+\tan n \cos \lambda \sin \theta+\tan n \cos \lambda \sin \theta+\cos \theta=0 .
\end{aligned}
$$

The integrals of the characteristic system of the first equations are

$$
\varphi, \quad D=\cos n \sin \lambda, \quad E=\theta-\arcsin \left(\sin n\left(1-D^{2}\right)^{-1 / 2}\right), \quad I .
$$


The latter integral can be represented in other forms

$$
\begin{aligned}
& \sin \theta \cos n \cos \lambda-\cos \theta \sin n=\sqrt{1-D^{2}} \sin E=K, \\
& \cos \theta \cos n \cos \lambda+\sin \theta \sin n=\sqrt{1-D^{2}} \cos E=\tilde{K} .
\end{aligned}
$$

We represent the general solution of the first equation in system $(2.10)$ as $K=G(\varphi, I, D)$. Then the second equation in system (2.10) casts into the form

$$
G_{\varphi}-G_{D} \sqrt{1-K^{2}-G^{2}}=0 .
$$

This implies that $K$ is the integral of the characteristic equation of second equation in system (2.10). The general solution of (2.11) reads as

$$
K=G\left(I, \varphi+\arcsin \frac{D}{\sqrt{1-K^{2}}}\right),
$$

where $m(K, I)$ is an arbitrary function. If $G_{D}=0$, then $G_{\varphi}=0$.

We write the general solution of system (2.6), (2.7) in an implicit form:

$$
\begin{gathered}
\cos n \sin \lambda=\sqrt{1-K^{2}} \sin (m(K, I)-\varphi), \\
K=\cos n \sin \theta \cos \lambda-\cos \theta \sin n, \quad I=t-\int U^{-1} d r, \quad n^{\prime}=H_{0} r^{-2} U^{-1},
\end{gathered}
$$

or $K=G(I)$ with an arbitrary function $G(I)$.

\section{Simple IRREGULAR PARTIALLY INVARIANT SOLUTIONS}

Subalgebras 4.40 and 4.34 define an irregular partially invariant solution of rank 1 defect 1 called a simple wave in gas dynamics [4]. The level sets of invariant functions are planes.

Let us consider an self-similar simple wave on sublagebra 4.26. The invariants determine the representation of solution

$$
\vec{u}=t^{-1} \vec{x}+\vec{u}_{1}(\alpha), \quad p=p(\alpha), \quad \rho=\rho(\alpha), \quad \alpha=\alpha(t, \vec{x}) .
$$

Equations (2.1) cast into the form

$$
\begin{aligned}
& \vec{u}_{1}^{\prime}\left(D \alpha+t^{-1} \vec{x} \cdot \nabla \alpha\right)+t^{-1} \vec{u}_{1}+\rho^{-1} p^{\prime} \nabla \alpha=0, \\
& \rho^{\prime}\left(D \alpha+t^{-1} \vec{x} \cdot \nabla \alpha\right)+\rho\left(\vec{u}_{1}^{\prime} \cdot \nabla \alpha+3 t^{-1}\right)=0, \\
& S^{\prime}\left(D \alpha+t^{-1} \vec{x} \cdot \nabla \alpha\right)=0, \quad p=f(\rho, S),
\end{aligned}
$$

where $D=\partial_{t}+\vec{u}_{1} \cdot \nabla$. If $S^{\prime} \neq 0$, then $p^{\prime} \neq 0$,

$$
\nabla \alpha=-\frac{\rho}{t p^{\prime}} \vec{u}_{1} \quad \Rightarrow \quad \vec{u}_{1}^{\prime} \times \vec{u}_{1}=0 \quad \Rightarrow \quad \vec{u}_{1}^{\prime}=\beta(\alpha) \vec{u}_{1} ; \quad \alpha_{t}=\frac{\rho}{t p^{\prime}}\left(t^{-1} \vec{x}+\vec{u}_{1}\right) \cdot \vec{u}_{1} .
$$

The compatibility leads us to the condition $\beta=0, \vec{u}_{1}=\vec{u}_{0}$ is a constant invariant velocity. Then for the enthalpy and velocity we obtain the formulae

$$
i=\int \rho^{-1} d p=-t^{-1} \vec{u}_{0} \cdot \vec{x}+\vec{u}_{0}^{2} \ln |t|+i_{0}, \quad \vec{u}=t^{-1} \vec{x}+\vec{u}_{0} .
$$

At that, the entropy and enthalpy are arbitrary functions of the parameter $\alpha$. The level set of invariant functions is a plane with the normal vector $\vec{u}_{0}$ moving in the direction of the normal with variable velocity and acceleration.

In isentropical motion $S=S_{0}$, system (3.1) consists of two equations

$$
\begin{aligned}
& \alpha_{t}=-3 \rho\left(t \rho^{\prime}\right)^{-1}-\left(\vec{u}_{1}+t^{-1} \vec{x}+\rho\left(\rho^{\prime}\right)^{-1} \vec{u}_{1}^{\prime}\right) \cdot \nabla \alpha, \\
& \rho\left(\rho^{\prime}\right)^{-1} \vec{u}_{1}^{\prime}\left(3 t^{-1}+\vec{u}_{1}^{\prime} \cdot \nabla \alpha\right)=t^{-1} \vec{u}_{1}+p^{\prime} \rho^{-1} \nabla \alpha .
\end{aligned}
$$

We calculate the scalar product of the second equation with $\vec{u}_{1}^{\prime}$ and we get

$$
\vec{u}_{1}^{\prime} \cdot \nabla \alpha\left(\vec{u}_{1}^{2}-p^{\prime} \rho^{\prime} \rho^{-2}\right)=t^{-1}\left(\rho^{\prime} \rho^{-1} \vec{u} \cdot \vec{u}_{1}^{\prime}-3 \vec{u}_{1}^{\prime 2}\right) .
$$


As $\vec{u}_{1}^{2} \neq p^{\prime} \rho^{\prime} \rho^{-2}$, we obtain

$$
\begin{aligned}
& \nabla \alpha=t^{-1} \vec{A} \Rightarrow \overrightarrow{A^{\prime}} \times \vec{A}=0 \Rightarrow \vec{A}=\vec{A}_{0} m^{\prime}(\alpha)^{-1}, \quad m(\alpha)=t^{-1} \vec{A}_{0} \cdot \vec{x}, \\
& \alpha_{t}=-t^{-1}\left(3 \rho \rho^{-1}+\left(\vec{u}_{1}+t^{-1} \vec{x}+\rho \rho^{-1} \vec{u}_{1}^{\prime}\right) \cdot \vec{A}\right) \quad \Rightarrow \quad 3 \rho+(\rho \kappa)^{\prime}=0,
\end{aligned}
$$

where $\kappa=\vec{A}_{0} \cdot \vec{u}_{1}, \vec{u}_{1}^{2} \neq p^{\prime} \rho^{\prime} \rho^{-2}$,

$$
\vec{A}=-\frac{\rho}{p^{\prime}} \vec{u}_{1}+\vec{u}_{1}^{\prime} \frac{\rho p^{\prime-1} \vec{u}_{1} \cdot \vec{u}_{1}^{\prime}-3}{\vec{u}_{1}^{\prime 2}-p^{\prime} \rho^{\prime} \rho^{-2}} .
$$

We can assume $m^{\prime}=1$. The level set is a plane with the normal $\vec{A}_{0}$. The compatibility conditions cast info the form

$$
\overrightarrow{A_{0}}\left(\frac{\rho^{\prime}}{\rho}-\frac{\rho^{\prime \prime}}{\rho^{\prime}}+\frac{\rho^{\prime}}{3 \rho}\left(\vec{u}_{1}+\frac{\rho}{\rho^{\prime}}\right)^{\prime} \cdot \vec{A}\right)=0 .
$$

If $\vec{A}=0$, then (3.3) implies the solution of system (3.1):

$$
\alpha=t, \quad \rho=\rho_{0} t^{-3}, \quad \vec{u}_{1}=\vec{u}_{0} t^{-1}, \quad p=f\left(S_{0}, \rho_{0} t^{-1}\right) .
$$

As $\overrightarrow{A_{0}} \neq 0$, we calculate the scalar product of (3.4) with $\overrightarrow{A_{0}}$ :

$$
\left(\kappa^{\prime}+3\right) \vec{u}_{1}^{\prime 2}=\rho^{-1} \rho^{\prime}\left(p^{\prime} \rho^{-1} \kappa^{\prime}+\vec{u}_{1} \cdot \vec{u}_{1}^{\prime}\right) .
$$

Then identities (3.4) become:

$$
\left(\vec{u}_{1} \cdot \vec{u}_{1}^{\prime}-3 \rho^{-1} p^{\prime}\right)\left(\left(p^{\prime} \rho^{-1} \vec{A}_{0}+\vec{u}_{1}\right) \rho^{\prime} \rho^{-1}-\vec{u}_{1}^{\prime}\left(\kappa^{\prime}+3\right)\right)=0 .
$$

If we vanish the first factor, then by (3.4), (3.3) we obtain the solution

$$
\vec{u}_{1}=-\vec{A}_{0} A_{0}^{-2} \alpha, \quad \alpha=t^{-1} \vec{A}_{0} \cdot \vec{x}, \quad \rho=\rho_{0} \alpha^{2}, \quad p=p_{0}+\frac{3 \rho_{0}}{4 A_{0}^{2}} \alpha^{4} .
$$

By vanishing the second factor, we obtain the solution:

$$
\frac{\rho^{\prime}}{\rho}=-\frac{\kappa^{\prime}+3}{\kappa}, \quad A_{0}^{2} \frac{p^{\prime}}{\rho}=-\kappa\left(\kappa \kappa^{\prime}+1\right), \quad \vec{u}_{1}=A_{0}^{-2} \vec{A}_{0} \kappa+\vec{B}_{0} \exp \left(-\int \kappa^{-1} d \alpha\right) .
$$

It remains to consider the case $\vec{u}_{1}^{2}=p^{\prime} \rho^{\prime} \rho^{-2}$. It is convenient to introduce the enthalpy $i=\int \rho^{-1} d p$ and to replace the parameter $\alpha$ by the density $\rho(\alpha)$. System of equations (3.1) becomes

$$
\begin{aligned}
& \rho \vec{u}_{1 \rho}^{2}=i_{\rho}, \quad \vec{u}_{1}^{2}=6 i, \quad \vec{u}_{1} \cdot \vec{u}_{1 \rho}=3 i_{\rho}, \\
& \rho \vec{u}_{1 \rho}\left(3 t^{-1}+\vec{u}_{1 \rho} \cdot \nabla \rho\right)=t^{-1} \vec{u}_{1}+i_{\rho} \nabla \rho, \\
& \rho_{t}+\left(\vec{u}_{1}+\rho \vec{u}_{1 \rho}+t^{-1} \vec{x}\right) \cdot \nabla \rho=-3 t^{-1} \rho .
\end{aligned}
$$

The change of variables

$$
\vec{X}=t^{-1} \vec{x}, \quad t^{3} \rho=R(t, \vec{X})
$$

reduces system (3.9) to a simple form

$$
\begin{aligned}
& R_{t}+\left(R \vec{u}_{1}\right)_{R} \cdot \nabla R=0, \quad i_{R} \nabla R+\vec{u}_{1}=R \vec{u}_{1 R}\left(3+\vec{u}_{1 R} \cdot \nabla R\right), \\
& R \vec{u}_{1 R}^{2}=i_{R}, \quad \vec{u}_{1}^{2}=6 i, \quad \vec{u}_{1} \cdot \vec{u}_{1 R}=3 i_{R} .
\end{aligned}
$$

The compatibility conditions of a vector equation in (3.10) is the vanishing of a triple product:

$$
\left(\vec{u}_{1 R R}, \vec{u}_{1 R}, \vec{u}_{1}\right)=0
$$

which is equivalent to the system of equations

$$
\vec{u}_{1 R R}=a \vec{u}_{1 R}+b \vec{u}_{1}, \quad R \vec{u}_{1 R}^{2}=i_{R}, \quad \vec{u}_{1}^{2}=6 i,
$$

where

$$
a=\frac{i_{R R}\left(i-3 R i_{R}\right)+\left(i_{R}-R^{-1} i\right) i_{R}}{i_{R}\left(2 i-3 R i_{R}\right)}, \quad b=\frac{i i_{R R}+3^{-1} R^{-1} i_{R}}{2\left(2 i-3 R i_{R}\right)} .
$$


We substitute the general solution of the first equation in system (3.10)

$$
R=\varphi(\vec{I}), \quad \vec{I}=\vec{X}-t\left(R \vec{u}_{1}\right)_{R}
$$

into the second vector equation

$$
i_{R} \nabla \varphi-R \vec{u}_{1 R}\left(\vec{u}_{1 R} \cdot \nabla \varphi\right)=\left(3 R \vec{u}_{1 R}-\vec{u}_{1}\right)\left(1+t \nabla \varphi \cdot\left(\vec{u}_{1} R\right)_{R R}\right) .
$$

Here $t$ is a free variable, the sought function $\varphi$ is independent of this variable. Thus, we can vanish the coefficient at $t$ and to obtain the compatibility condition of system (3.10)

$$
\nabla \varphi \cdot\left(\vec{u}_{1} R\right)_{R R}=0 \quad \Rightarrow \quad \nabla R \cdot\left(\vec{u}_{1} R\right)_{R R}=0 .
$$

The scalar product of the vector equation in (3.10) with $\vec{u}_{1}$ gives one more equation:

$$
\vec{u}_{1 R} \cdot \nabla R=\frac{3 R b\left(2 i-3 R i_{R}\right)}{i_{R}\left(3 R^{2} b+R a+2\right)}=\beta, \quad \vec{u}_{1} \cdot \nabla R=-\beta \frac{2+R a}{R b} .
$$

We substitute these expressions into (3.10) and we arrive at a compatible system of identities:

$$
R_{t}=\beta(R b)^{-1}\left(2+R a-R^{2} b\right)=N(R), \quad \nabla R=i_{R}^{-1}\left(-\vec{u}_{1}+R \vec{u}_{1 R}(3+\beta)\right)=\vec{n}_{0} e^{k(R)},
$$

where $\vec{n}_{0}$ is a constant vector. The general solution of each equation is of the form

$$
\int N(R)^{-1} d R=t+f(\vec{x}), \quad \int e^{-k(R)} d r=\vec{n}_{0} \cdot \vec{X}+m(t) .
$$

Since these identities determine the same solution, we have

$$
f(\vec{X})=\vec{n}_{0} \cdot \vec{X}, \quad m(t)=t, \quad N=e^{k(R)} .
$$

Thus, we have obtained the solution:

$$
\int e^{-k(R)} d R=t+\vec{n}_{0} \cdot \vec{X}
$$

with an arbitrary function $i(\rho)$ and the invariant velocity obeying (3.11).

Let us consider a stationary simple wave on subalgebra 4.10. The invariants provide the representation of the solution in the cylindrical coordinates:

$$
U=U(\alpha), \quad q_{C}=q(\alpha), \quad \vartheta_{C}=x-\theta+\vartheta(\alpha), \quad p=p(\alpha), \quad \rho=\rho(\alpha), \quad \alpha=\alpha(t, \vec{x}) .
$$

System (2.2) becomes:

$$
\begin{aligned}
& U^{\prime} D \alpha+\rho^{-1} p^{\prime} \alpha_{x}=0, \quad q^{\prime} D \alpha+\rho^{-1} p^{\prime} \partial_{c} \alpha=0, \quad q\left(\vartheta^{\prime} D \alpha+U\right)+\rho^{-1} p^{\prime} \partial_{s} \alpha=0, \\
& \rho^{\prime} D \alpha+\rho\left(U^{\prime} \alpha_{x}+q^{\prime} \partial_{c} \alpha+q \vartheta^{\prime} \partial_{s} \alpha\right)=0, \quad S^{\prime} D \alpha=0,
\end{aligned}
$$

where

$$
D=\partial_{t}+U \partial_{x}+q \partial_{c}, \quad \partial_{c}=\cos \vartheta_{C} \partial_{r}+r^{-1} \sin \vartheta_{C} \partial_{\theta}, \quad \partial_{s}=-\sin \vartheta_{C} \partial_{r}+r^{-1} \cos \vartheta_{C} \partial_{\theta} .
$$

Let $S^{\prime} \neq 0, p^{\prime}=0$. Then $D \alpha=0, q U=0$. As $U=0$, by (3.12) we get an overdetermined system

$$
\alpha_{t}+q \partial_{c} \alpha=0, \quad q^{\prime} \partial_{c} \alpha+q \vartheta^{\prime} \partial_{s} \alpha=0
$$

We substitute the general solution of the first equation in system (3.13)

$$
q t+r \cos \vartheta_{C}+\varphi(x, \alpha, I), \quad I=r \sin \vartheta_{C}
$$

into the second equation in system (3.13)

$$
q^{\prime}=q \vartheta^{\prime} \varphi_{I} \quad \Rightarrow \quad \varphi=q^{\prime}\left(q \vartheta^{\prime}\right)^{-1} I+\psi(x, \alpha) .
$$

Thus, the general solution of (3.13) reads as

$$
q t=r \cos \vartheta_{C}+q^{\prime}\left(q \vartheta^{\prime}\right)^{-1} r \sin \vartheta_{c}+\psi(x, \alpha), \quad \vartheta_{c}=x-\theta+\vartheta(\alpha) .
$$

As $q=0$, system (3.12)

$$
\alpha_{t}+U \alpha_{x}=0, \quad U^{\prime} \alpha_{x}=0
$$


has a solution

$$
U=U_{0}, \quad \alpha=\alpha\left(x-U_{0} t, r, \theta\right)
$$

or

$$
\alpha=\alpha(r, \theta) \text { for } \quad U^{\prime} \neq 0 .
$$

As $S^{\prime} \neq 0, p^{\prime} \neq 0$, system (3.12) is incompatible.

Let $S^{\prime}=0, U^{\prime} \neq 0, i^{\prime}=\rho^{-1} p^{\prime} \neq 0$. Then it follows from (3.12) that

$$
\begin{aligned}
& \alpha_{x}=A(\alpha)=q^{2} U U^{\prime} \vartheta^{\prime} i^{\prime-1}\left(U^{\prime 2}+q^{\prime 2}+q^{2} \vartheta^{\prime 2}-\rho^{-1} \rho^{\prime} i^{\prime}\right)^{-1}, \quad \alpha_{t}=-k(\alpha) A, \\
& k(\alpha)=U+U^{\prime-1}\left(i^{\prime}+q q^{\prime}\right), \quad \partial_{c} \alpha=q^{\prime} U^{\prime-1} A, \quad \partial_{s} \alpha=q \vartheta^{\prime} U^{\prime-1} A-q U i^{\prime-1} .
\end{aligned}
$$

The compatibility condition leads us to the identity $A k^{\prime}=0$. The case $A=0$ gives rise to a contradiction. As $k=k_{0}$, where $k_{0}$ is constant, the Bernoulli integral holds:

$$
U^{2}+q^{2}+2 i=2 k_{0} U+B_{0} .
$$

If $\vartheta^{\prime}=0$, there are no solutions. As $\vartheta^{\prime} \neq 0$, system (3.15) casts into the form:

$$
\begin{aligned}
& \alpha=\alpha(s, r, \theta), \quad s=x-k_{0} t, \quad \alpha_{s}=A(\alpha), \quad \vartheta_{C}=s-\theta+\vartheta+k_{0} t, \\
& q^{\prime} U^{\prime-1} A=\partial_{c} \alpha, \quad q\left(-U i^{\prime-1}+\vartheta^{\prime} U^{\prime-1} A\right)=\partial_{s} \alpha .
\end{aligned}
$$

By differentiating in $t$ we get

$$
k_{0} \partial_{s} \alpha=0, \quad k_{0} \partial_{c} \alpha=0 .
$$

As $k_{0} \neq 0$, we obtain the solution

$$
q=q_{0} \neq 0, \quad i^{\prime}=U^{\prime}\left(k_{0}-U\right), \quad \rho\left(k_{0}-U\right)=\rho_{0}, \quad k_{0} \int U^{-1} d \vartheta-\vartheta=x-k_{0} t .
$$

As $k_{0}=0$, we obtain the relations:

$$
\begin{aligned}
& \vartheta_{C}=x-\theta+\vartheta(\alpha), \quad \alpha_{t}=0, \quad \int A^{-1} d \alpha=x+\varphi(r, \theta), \\
& q^{\prime} U^{\prime-1}=\partial_{C} \varphi, \quad q A^{-1}\left(-U i^{\prime-1}+\vartheta^{\prime} A U^{\prime-1}\right)=\partial_{S} \varphi .
\end{aligned}
$$

Differentiating (3.17) in $x$, we get the compatibility equations:

$q^{\prime} U^{\prime-1}=a_{0} \sin \sigma(\alpha), \quad q A^{-1}\left(U i^{\prime-1}-\vartheta^{\prime} A U^{\prime-1}\right)=a_{0} \cos \sigma(\alpha), \quad \sigma+\vartheta=-\int A^{-1} d \alpha=-x-\varphi$.

Equations (3.17) are rewritten as

$$
-a_{0} \cos \varphi=\sin \theta \varphi_{r}+r^{-1} \cos \theta \varphi_{\theta}, \quad-a_{0} \sin \varphi=\cos \theta \varphi_{r}-r^{-1} \sin \theta \varphi_{\theta} .
$$

In the Cartesian coordinates $y=r \cos \theta, z=r \sin \theta$, the equations become

$$
\varphi_{y}=-a_{0} \sin \varphi, \quad \varphi_{z}=-a_{0} \cos \varphi
$$

The compatibility condition is $a_{0}=0$. Hence, $\varphi=0, q^{\prime}=0, U^{\prime 2}=\rho^{-1} \rho^{\prime} i^{\prime}$, and the solution

$$
\int \rho \vartheta^{\prime} U^{\prime} U^{-1} \rho^{\prime-1} d \alpha=x
$$

is reduced to the invariant one on sublagebra 3.33 [4].

The case $U^{\prime} \neq 0, p^{\prime}=0, S^{\prime}=0$ is reduced to a one-dimensional plane isobaric wave

$$
p=p_{0}, \quad S=S_{0}, \quad V=W=0, \quad x-t U=F(U, r, \theta) .
$$

The case of constant $S=S_{0}, U=U_{0}, p=p_{0}$ gives the solution

$$
q_{0} t+\psi(x, x+\vartheta)=r \cos (x+\vartheta-\theta),
$$

where $q=q_{0}$ is a constant, $\psi(x, I)$ is an arbitrary function. In the case $S=S_{0}, p=p_{0}, q^{\prime} \neq 0$, $U=0$ we have an overdetermined system

$$
\alpha_{t}+q \partial_{c} \alpha=0, \quad q^{\prime} \partial_{c} \alpha+q \vartheta^{\prime} \partial_{s} \alpha=0 .
$$


The general solution of the latter equation is of the form

$$
r\left(q^{\prime} \sin \vartheta_{C}+q \vartheta^{\prime} \cos \vartheta_{C}\right)=\varphi(t, x, \alpha) .
$$

The first equation determines the function $\varphi$

$$
\varphi_{t}=q^{2} \vartheta^{\prime} \Rightarrow \varphi=q^{2} t \vartheta^{\prime}+\psi(x, \alpha) .
$$

We obtain the solution in the explicit form $U=0$,

$$
r\left(q^{\prime} \sin (x-\theta+\vartheta)+q \vartheta^{\prime} \cos (x-\theta+\vartheta)\right)=q^{2} t \vartheta^{\prime}+\psi(x, \alpha),
$$

where $q(\alpha), \vartheta(\alpha)$ are arbitrary functions, $p_{0}=f\left(\rho_{0}, S_{0}\right)$. This is a solutions with two arbitrary functions $q(\alpha)$ and $\psi(x, \vartheta)$. The case $S=S_{0}, U=U_{0}, p^{\prime} \neq 0$ leads us to a contradiction and the absence of solution.

Thus, on subalgebra 4.10 we obtain a series of simple waves given by formulae (3.14), (3.16), (3.18), (3.20).

\section{CONCLUSion}

Simple solutions on 4-dimensional subalgebras are regular and irregular partially invariant solutions of rank 1 defect 1 . To classify them, we have calculated the bases of point invariants for all 48 classes of subalgebras in the optimal system of non-similar subagebras of 11-dimensional Lie algebra admitted by the equations of continuous medium mechanics. For a hydrodynamics model we have found new simple waves for three subalgebras with regular partially invariant solutions and for two-subalgebras with irregular partially invariant solutions.

\section{BIBLIOGRAPHY}

1. S.V. Khabirov, Yu.A. Chirkunov. Emelents of symmetry analysis of differential equations of contionuous media mechanics. Novosibirsk State Tech. Univ., Novosibirsk (2012). (in Russian).

2. L.V. Ovsyannikov. The "PODMODELI" program. Gas dynamics // Prikl. matem. i mekh. 58:4, 30-55 (1994). [J. Appl. Math. Mech. 58:4, 601-627 (1994).]

3. E.V. Mamontov. Invariant submodels of rank two to the equations of gas dynamics // Prikl. Mekh. Tekh. Fiz. 40:2, 50-55 (1999). [J. Appl. Mech. Tech. Phys. 40:2, 232-237 (1999).]

4. S.V. Khabirov. Lectures on analytic methods in gas dynamics. Bashkir State Univ., Ufa (2013). (in Russian).

5. L.V. Ovsyannikov. "Simple" solutions to the dynamics equations for a polytropic gas // Prikl. Mekh. Tekh. Fiz. 40:2, 5-12 (1999).[J. Appl. Mech. Tech. Phys. 40:2, 191-197 (1999).]

6. S.V. Khabirov. A hierarchy of submodels of differential equations// Sibir. Matem. Zhurn. 54:6, 1396-1406 (2013). [Siber. Math. J. 54:6, 1111-1120 (2013).]

7. L.V. Ovsyannikov. Regular and nonregular partially invariant solutions // Dokl. Akad. Nauk. 343:2, 156-159 (1995). [Dokl. Math. 52:1, 23-26 (1995).]

8. A.A. Cherevko, A.P. Chupakhin. Ovsyannikov stationary vortex // Preprint 1-2005, Lavrentiev Hydrodanymics Inst., Novosibirsk (2005). (in Russian).

Salavat Valeevich Khabirov,

Mavlyutov Institute of Mechanics,

Ufa Federal Research Center, RAS,

Oktyabr' av. 71,

450054, Ufa, Russia

E-mail: habirov@anrb.ru 